\title{
Ambiguity function display using a single 1-D input
}

Robert J. Marks II and Michael W. Hall

University of Washington, Department of Electrical Engineering, Seattle, Washington 98195.

Received 12 April 1979.

0003-6935/79/152539-02\$00.50/0.

(C) 1979 Optical Society of America.

The ambiguity function, first introduced by Woodward, ${ }^{1}$ is a measure of the capability of a signal to measure the range and velocity of a target. The range is determined by time delay $\tau$ and the velocity by Doppler shift $\nu$. The ambiguity function is defined as

$$
\chi(\nu ; \tau)=\int_{-\infty}^{\infty} u(t) u(t-\tau) \exp (-j 2 \pi \nu t) d t,
$$

where we have assumed $u(t)$ is real.

A coherent processor capable of displaying the ambiguity function over the entire $(\nu, \tau)$ plane has been presented..$^{2,3}$ This Letter outlines an alternate scheme for ambiguity function display with the advantage that only a single $1-D$ input is required. This eliminates the problem of maintaining identical generation and strict alignment of two versions of the same input. There are other bilinear coherent processors that require two identical 1-D inputs. ${ }^{4}$ The technique described herein can also be straightforwardly applied to these processors, so that only a single 1-D input is needed. 


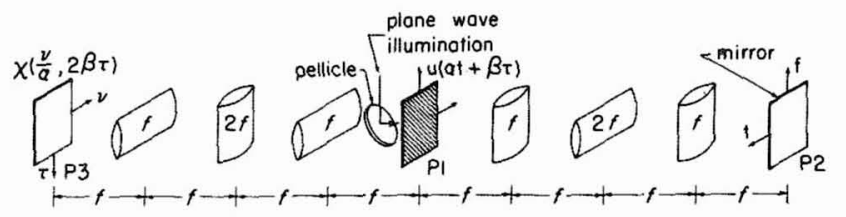

Fig. 1. A coherent processor for ambiguity-function display using a single 1-D input.
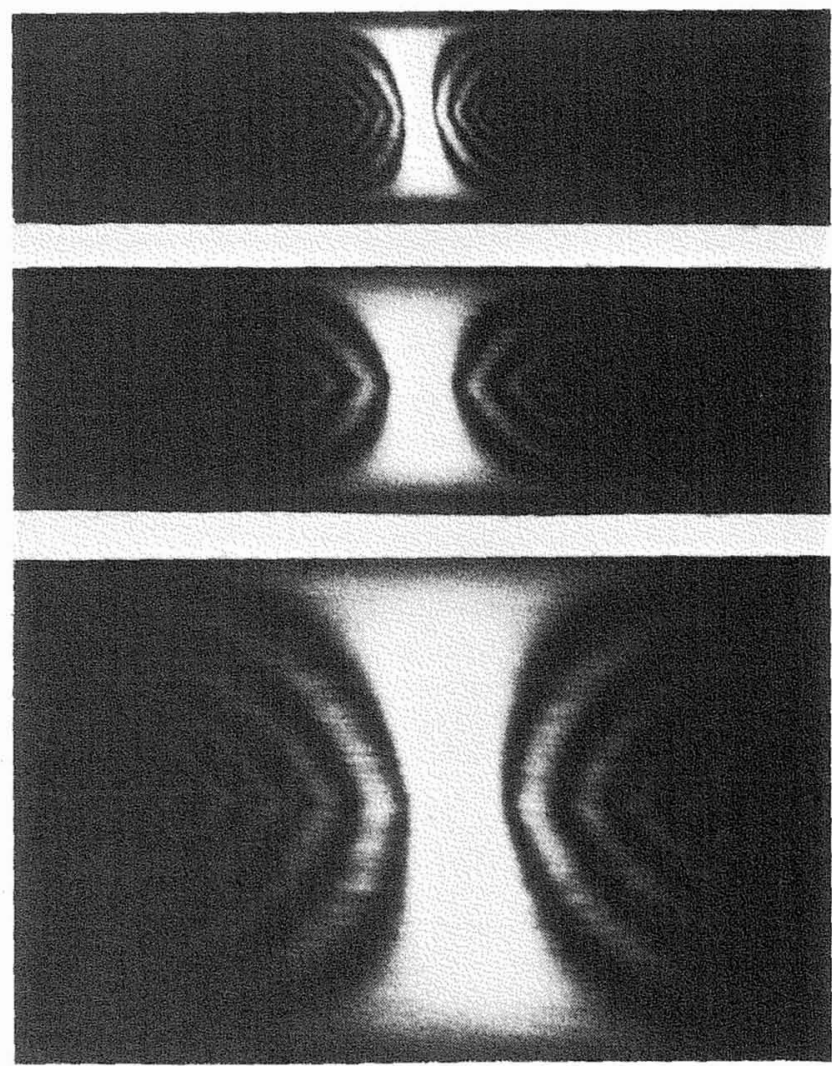

Fig. 2. The ambiguity-function display for a single square pulse for three values of $\theta$.

The single-input ambiguity-function processor is pictured in Fig. 1. Coherent illumination is introduced into the system via a pellicle. The 1-D input $u(t)$ is rotated an angle of $\theta$ on the $(t, \tau)$ plane to yield an input transmittance of $u(\alpha t+\beta \tau)$, where $\alpha=\cos \theta$ and $\beta=\sin \theta$. To the right of the input is an astigmatic processor. ${ }^{5}$ Disregarding proportionality constants (as we shall do henceforth), the field amplitude incident on plane $P 2$ is

$$
\int_{-\infty}^{\infty} u(\alpha t+\beta \tau) \exp (-j 2 \pi f \tau) d \tau
$$

where the spatial frequency $f$ is measured by dividing the vertical displacement on plane $P 2$ by $2 \lambda f .^{5}$ Lambda is the wavelength of the spatially coherent illumination, and $2 f$ is the focal length of the center cylindrical lens.

On plane $P 2$ we place a mirror which reflects the field am. plitude in Eq. (2) back into the astigmatic processor. As can be shown by a second application of Eq. (2), the net result is that the field amplitude incident on plane $P 1$ from the right is $u(\alpha t-\beta \tau)$. That is, upon going through the astigmatic processor twice, the input undergoes a coordinate reversal in $\tau$.
The field amplitude which exits plane $P 1$ from the right is $u(\alpha t+\beta \tau) u(\alpha t-\beta \tau)$. This is input into the left-hand astigmatic processor through the pellicle. Thus, we have incident on plane $P 3$

$$
\begin{aligned}
\int_{-\infty}^{\infty} u(\alpha t & +\beta \tau) u(\alpha t-\beta \tau) \exp (-j 2 \pi \nu t) d t \\
= & \frac{1}{\alpha} \exp (j 2 \pi \nu \tau \beta / \alpha) \int_{-\infty}^{\infty} u(\hat{t}) u(\hat{t}-2 \beta \tau) \exp (-j 2 \pi \nu \hat{t} / \alpha) d \hat{t} \\
& =\frac{1}{\alpha} \exp (j 2 \pi \nu \tau \beta / \alpha) \chi\left(\frac{\nu}{\alpha}, 2 \beta \tau\right)
\end{aligned}
$$

where in the second step we have made the variable substitution $\hat{t}=\alpha t+\beta \tau$. The spatial frequency $\nu$ is measured in the same manner as $f$.

Outside of the phase term, we see from Eq. (3) that a scaled version of the ambiguity function is incident on plane $P 3$. In most cases, it is the squared modulus of the ambiguity function which is of interest. This corresponds to the intensity distribution on plane P3. Thus, in most cases. the phase term in Eq. (3) is of no concern. Note that scaling of the output can be achieved by a simple rotation of the input.

The disadvantages of this processor compared to the double-input processor are (1) less efficient utilization of incident light due to pellicle loss, (2) increased optics in the beam path, and (3) the inability to perform cross-ambiguity-function operations. These negative aspects, however, are offset by the fact that only a single 1-D input is used in this processor.

To illustrate the performance of the single-input processor, the ambiguity function of a single square pulse was generated for three values of $\theta$. The results, pictured in Fig. 2, compare quite favorably to the corresponding double-input results of Ref. 3.

This work was generously supported by the University of Washington Graduate Research Fund, Project PSE-517.

\section{References}

1. P. W. Woodward, Probability and Information Theory With Applications to Radar (Pergamon, Oxford, 1963).

2. R. A. K. Said and D. C. Cooper, Proc. Inst. Electr. Eng. 120, 423 (1973).

3. R. J. Marks II, J. F. Walkup, and T. F. Krile, Appl. Opt. 16, 746 (1977); addendum 16, 1777 (1977).

4. B. E. A. Saleh, Appl. Opt. 17, 3408 (1978).

5. R. J. Marks II and S. V. Bell, Opt. Eng. 17, 167 (1978). 